\title{
Entrelacs
}

Cinéma et audiovisuel

\section{Paris déserté par le regard. Note pour un film transhistorique : Arc Arceaux Arcades}

Laurent Roth

\section{(2) OpenEdition}

\section{Journals}

Édition électronique

URL : https://journals.openedition.org/entrelacs/6183

DOI : 10.4000 /entrelacs.6183

ISSN : 2261-5482

Éditeur

Éditions Téraèdre

Référence électronique

Laurent Roth, «Paris déserté par le regard. Note pour un film transhistorique : Arc Arceaux Arcades », Entrelacs [En ligne], 18 | 2021, mis en ligne le 13 décembre 2021, consulté le 15 décembre 2021. URL http://journals.openedition.org/entrelacs/6183; DOI : https://doi.org/10.4000/entrelacs.6183

Ce document a été généré automatiquement le 15 décembre 2021.

Tous droits réservés 


\title{
Paris déserté par le regard. Note pour un film transhistorique : Arc Arceaux Arcades
}

\author{
Laurent Roth
}

«Une de nos tâches les plus urgentes est de réapprendre à voyager, éventuellement au plus proche de chez nous, pour réapprendre à voir... »

Marc Augé, Un Ethnologue dans le métro ${ }^{1}$ « Comment décrire Paris sans faire référence à la "carte postale" qui a fait l'émerveillement des spectateurs de Midnight in Paris récemment? Comment la traverser, la parcourir, l'évoquer, la représenter sans succomber à l'angoisse de la réification, de la fossilisation, à la peur de la pétrification? Comment sauver l'éphémère, le fugitif sans le figer? Comment éviter, en un mot, la destruction de la ville par son image même?" Régine Robin, Le Mal de Paris ${ }^{2}$

chantier du Centre Georges Pompidou, j'ai vu la ville monde rayonner sur la planète sans réussir à résorber en son sein inégalités et exclusions.

4 Paris perd ses habitants : les jeunes, les vieux, les familles nombreuses, les ouvriers, les artistes, les intellectuels et ce qu'on appelle les classes moyennes ont de plus en plus de mal à y habiter ou y faire un projet de vie.

J'ai depuis très longtemps envie de filmer Paris.

Je palpe avec anxiété le cœur d'une ville qui se refroidit.

Paris se pétrifie, se fossilise, se muséalise, en son cœur en tout cas. Dans ses extrémités, le sang coule encore, mais pour combien de temps? 
6 À la faveur d'une résidence au Centquatre, j'ai vécu un an et demi au cœur du quartier de La Villette, un des quartiers les plus populaires de Paris, quartier qui n'intéresse aucun

touriste

et pourtant vrai laboratoire de ce que pourrait être l'intégration de la banlieue à la métropole.

7 Encombré de réseaux, canaux, entrepôts, voies ferrées, voies rapides et tours d'habitations, ce quartier Ouest de la Villette que j'ai arpenté depuis six ans avec le photographe Guillaume Vieira fait l'objet d'une préfiguration de ce que pourrait être le Grand Paris.

8 Avec le projet Paris Nord-Est, piloté par Dusapin et Leclerc, architectes urbanistes, ce sont 200 hectares à cheval sur les $18 \mathrm{e}$ et $19 \mathrm{e}$ arrondissements et les communes voisines de la Seine-Saint-Denis qui vont se trouver complètement remodelés, dans un secteur qui s'étire entre les Porte de la Chapelle et de la Villette, le long des boulevards extérieurs et du périphérique...

Pourtant, malgré ces projets grandioses, j'ai été frappé par ce sentiment de relégation que partagent les habitants du quartier, sentiment que l'on retrouve dans des banlieues plus lointaines, dont on ne parle que lorsque la rue flambe:comme à Noisiel ou Villiers-le-Bel, La Villette défraie la chronique lorsque ce sont les bandes rivales de la cité Riquet qui affrontent celle de la rue de Crimée...

Dans un périmètre volontairement restreint, j'ai donc choisi de faire la chronique de ce quartier, en me limitant à un pâté de maisons encadré par quatre rues : rue d'Aubervilliers, rue de Crimée, avenue de Flandre, boulevard de la Chapelle.

11 Tout comme le jeu de cubes utilisé comme test psychiatrique dans mon précédent film J'ai quitté l'Aquitaine, la figure de l'arc s'est imposée à moi comme un invariant insolite et pourtant plein de sens pour questionner l'espace urbain.

12 L'arc, l'arceau, l'arcade : figures de la " grande » architecture, religieuse, princière puis marchande, mais aussi principes urbains de la convivialité... On les retrouve partout pour qualifier l'espace du passage, qualifier aussi l'idée que l'on se fait des habitants, hier pour les inscrire dans la circulation marchande, aujourd'hui pour euphémiser la brutalité de l'urbanisme.

L'architecte dispose aujourd'hui des arcs dans la ville, dans un geste que l'on qualifiera de postmoderne, comme pour atténuer l'anonymat de l'espace urbain.

La thérapie imaginaire que j'ai inventée pour Arc Arceaux Arcades (abréviation: AAA) procède de la comptine, du mot d'esprit ou du jeu d'enfant : à travers un objet projectif et un quartier minuscule, raconter le tout de Paris et de son devenir... Walter Benjamin avait choisi les passages, Agnès Varda les cariatides, Chris Marker la jetée de l'aéroport d'Orly. Moi, je propose l'arc et ses variantes. On pourrait dire : chacun cherche son arche comme : chacun cherche son utopie.

Dans la narration de Arc Arceaux Arcades, je retrouve le personnage asilaire que j'avais créé dans mes deux précédents films, Une maison de famille et J'ai quitté l'Aquitaine. Ce personnage, candide et déplacé, a la même fonction que le Fou de la tragédie... Prêcher le faux pour obtenir le vrai, parler aux marges de l'imaginaire et de la société, faire écho à notre identité clivée et multiple.

16 La prétendue aliénation mentale de mon personnage lui permet, avec son partenaire médecin-chef Kendra Walker, de chevaucher les espaces et les époques librement, 
d'incarner plusieurs personnages à la fois, et surtout de faire rire (je l'espère), tout en délivrant des contenus savants, politiques et poétiques. C'est un griot qui raconte une autre histoire de France, tangentielle et minoritaire.

17 Les figures historiques qui s'invitent dans la narration de AAA ressemblent au quartier que je décris : elles sont reléguées, parfois oubliées, et pourtant si parlantes. Jacob Rodrigues Pereire, juif éclairé, premier instituteur des sourds-muets, qui achète un terrain rue de Flandre pour y créer le premier cimetière israélite de la capitale; le Maréchal Marmont, héros maudit de la bataille de Paris, qui signe la capitulation de l'Empire à la barrière des Vertus (Stalingrad) en mars 1814 ; ou encore Claude-Nicolas Ledoux, architecte utopiste des barrières de Paris, presque toutes détruites, sauf celle qui persiste sous le nom de " rotonde Stalingrad », et qui s'est imposée au cours de mes repérages comme l'œil du cyclone de mon film.

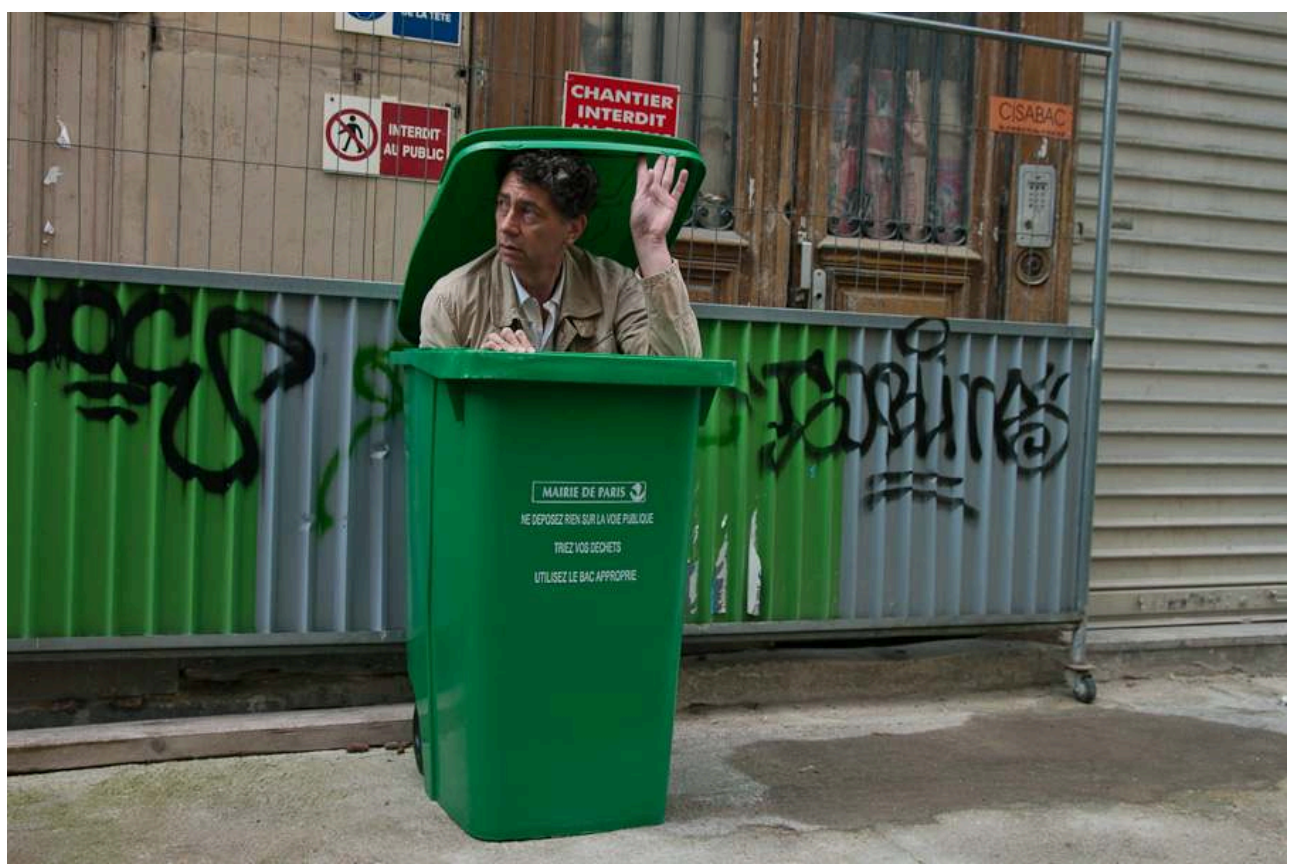

L'Emmuré de Paris : le patient - Diogène sort de sa poubelle. Photo non utilisée.

Crédit : La Huit, Guillaume Vieira.

À mi-chemin entre le réalisme noir et poétique d'Eli Lotar dans Aubervilliers et l'histrionisme fantaisiste et lettré de Sacha Guitry dans Si Paris m'était conté, j'aimerais tenter avec Arc Arceaux Arcades un nouvel essai de cinéma parisien transhistorique, à l'époque où, plus que jamais, se pose cette question taraudante : "Où est passé le peuple de Paris?»

Film fait seulement de voix, de sons et de photos, c'est un nouveau défi pour moi ! La photographie n'est pas ici un handicap, une forme de "cinéma diminué ». Sa fixité, bien au contraire, me semble révolutionnaire, pour indiquer, accuser, dénoncer ce danger de la pétrification qui menace Paris.

Tourné sur une longue échelle de temps, ce film est aussi un acte de foi dans le cinéma plus que jamais : inventer autrement le mouvement de ce qui a été, et du même coup, en faire un temps poétique, et qui sait... prophétique?

Novembre 2015. 


\section{NOTES}

1. [NDLR] Marc Augé, Un Ethnologue dans le métro, Paris, Hachette Littératures, 2008 [pagination non indiquée dans la note d'origine].

2. [NDLR] Régine Robin, Le Mal de Paris, Paris, Stock, 2014 [pagination non indiquée dans la note d'origine].

\section{RÉSUMÉS}

«Paris déserté par le regard» est la note d'intention écrite en novembre 2015 par le cinéaste Laurent Roth pour présenter son premier long métrage Arcs, Arceaux, Arcades. Le film, qui a trouvé sa forme définitive en 2021, s'intitule désormais L'Emmuré de Paris.

"Paris deserted by the gaze" is the note of intent written in November 2015 by filmmaker Laurent Roth to introduce his first feature film Arcs, Arceaux, Arcades. The film, which found its final form in 2021, is now entitled L'Emmuré de Paris.

\section{AUTEUR}

\section{LAURENT ROTH}

Après des études de philosophie, Laurent Roth profite de son service militaire en 1986 au sein de l'établissement cinématographique et photographique des armées pour réaliser Les Yeux brûlés un film qui est sorti en salles en 2015, après sa sélection au festival de Cannes, dans la section Cannes Classics. Suivront de nombreux courts et moyens métrages primés dans de grands festivals que La Huit a réunit dans un coffret paru en 2019. Depuis les années 2000, parallèlement à une activité d'écriture pour l'opéra et le théâtre, Laurent Roth poursuit son exploration des passerelles entre fiction et documentaire par une série de « fantaisies documentaires » où il interprète son propre personnage comme dans Une maison de famille (2004), J'ai quitté l'Aquitaine (2005) ou L'Emmuré de Paris (2021), son premier long métrage actuellement en post-production. Laurent Roth est également scénariste et a collaboré à l'écriture des films de Jean-Daniel Pollet, Vincent Dieutre, Dominique Cabrera ou encore Stéphane Batut. Comme critique de cinéma, il a notamment travaillé aux Cahiers du cinéma et pour France culture. Il a publié une centaine d'articles et d'essais, ainsi que deux livres consacrés à Abbas Kiarostami et à Chris Marker. Enfin, Laurent Roth a été pendant plusieurs années producteur au sein de la société Inthemood et directeur artistique de festivals (Festival International du documentaire « Fictions du réel » à Marseille, Ciné-citoyen à Paris). 